\title{
Prevalencia de felicidad en ciclos vitales y relación con redes de apoyo en población colombiana
}

\section{The prevalence of happiness in life-cycles and regarding support networks in the Colombian population}

Juan C. González-Quiñones y Guillermo Restrepo-Chavarriaga

Facultad de Medicina, Fundación Universitaria Juan N Corpas. Bogotá, Colombia. juan.gonzalez@juanncorpas.edu.co,guillermo.restrepo@juanncorpas.edu.co

Recibido 24 Julio 2009/Enviado para Modificación 13 Marzo 2010/Aceptado 25 Marzo 2010

\section{RESUMEN}

Objetivo Estimar la relación entre la felicidad y las redes de apoyo sociales.

Metodología Se realizó un estudio observacional de corte transversal entre 7335 personas repartidas en zona urbana (Bogotá) y rural (Ubaté) estratificadas por edades, por estratos (condición socioeconómica) y nivel de estudios. Se aplicó un cuestionario donde se interrogó por percepción del estado de ánimo del momento y por quién los apoya cuando tienen problemas.

Resultados Edad media de la población 31 años. El $31 \%$ se perciben felices. Dios es el mayor apoyo con prevalencia del $91 \%$, seguido por uno mismo con $77 \%$, amigo $60 \%$, madre $57 \%$, hermano con $50 \%$. Entre más red de apoyo, más felicidad con un OR de 6,6 (IC de $95 \%$; de 5-9).

Conclusión Fuerte relación entre felicidad y redes sociales de apoyo que favorecen la buena salud (bienestar) de las personas.

Palabras Clave: Felicidad, salud, desarrollo humano, relaciones interpersonales, relaciones familiares (fuente: DeCS, BIREME).

\section{ABSTRACT}

Objective Assessing the relationship between happiness and social support networks in Colombia.

Methodology A cross-sectional, observational study was carried out on 7,335 people living in an urban area (Bogotá) and a rural area (Ubaté) who had been stratified by age, economic status and last year of schooling. A questionnaire asked about the people's perception of their current mood and how they received support when they had problems. Results Average age was $31 ; 31 \%$ perceived that they were happy. God provided the greatest support ( $91 \%$ prevalence), followed by oneself $(77 \%)$, a friend $(60 \%)$, mother $(57 \%)$ and a brother/sister (50\%). The greater the support network, the greater the amount of perceived happiness (66 OR; 5-9 95\%Cl).

Conclusions $A$ strong relationship between happiness and social support networks promotes people's well being.

Key Words: Happiness, health, human development, interpersonal relationship, family relationship (source: MeSH, NLM). 
L

a visión de la salud en el ser humano, ha experimentado una transición desde el modelo de ausencia de la enfermedad hasta la búsqueda del estado de bienestar del individuo (OMS 1946). Bajo este último paradigma, la felicidad se convierte en un importante referente de bienestar (1), ello a pesar de la dificultad derivada de la determinación de medidas que permitan identificarla de manera precisa (2). Existe evidencia que las personas felices viven más, que tienen mayor propensión a estar sanas (3) y se defienden mejor de la enfermedad (4). Según estudios de diversos autores, la felicidad es una tendencia del estado de ánimo y en donde en común se encuentra que los individuos que se perciben felices (5) son seguros de sí mismos, emprendedores, optimistas y extrovertidos con la capacidad de mantener sólidas relaciones sociales con otros y comprometidos con la vida y los demás (6).

De esas relaciones sociales surge el concepto de redes de apoyo (7), el cual es interpretado en esta investigación como la ayuda que recibe el individuo de otros en situaciones adversas. El ejemplo más típico de apoyo al ser humano lo constituye la familia (8). Otros apoyos lo constituyen la pareja, los amigos (9), el vínculo a grupos sociales como, por ejemplo, los terapéuticos o grupos de tercera edad. Existen actividades propias del individuo que implican contacto con otros, tales como el trabajo o actividades de desarrollo religioso o las relacionadas con el estudio y que en común permiten contactos sociales (10) que pueden también generar importantes apoyos.

Las redes sociales del ser humano se establecen a lo largo de la vida, por tanto, apreciar la correspondencia entre estas y la felicidad puede ser útil para fortalecerlos y, al hacerlo, se promueve la salud de la población. El presente trabajo pretende estimar dichas relaciones y comportamientos de acuerdo a condiciones socioeconómicas (estratos), ubicación geográfica (urbana y rural) y acceso a la educación. Se plantea que esta descripción ayudará a "promover" las redes y con ello la felicidad y, así, construir estructuras sociales que sirvan para enfrentar las enfermedades.

\section{MATERIALES Y MÉTODOS}

Se trata de un estudio descriptivo de tipo transversal.

Población

Para la presente investigación se encuestaron personas en Bogotá (67 \%) y en el municipio de Ubaté (33\%) entre el 2002 y el 2003. La selección de la 
población se hizo con tres criterios: se estableció como condición que la rural (Ubaté) perteneciera a los estratos 1 y 2 (escala de 1 a 6 , expresando los más bajos un mayor nivel de pobreza) y la urbana (Bogotá) a los estratos 1 y 2, por el predominio de éstos en los barrios donde vivían (localidad de Suba). La población de estratos $3,4,5$ y 6 a través de conocidos de estudiantes de una facultad de medicina privada. La población fue escogida por su deseo a participar usando encuestadores preparados específicamente para aplicar el cuestionario (muestreo por conveniencia). El único criterio de exclusión fue la negativa a ofrecer la información. Se explicó el propósito del estudio, se aseguró la confidencialidad y se hicieron las preguntas. La población se estratificó por ciclo vital de la siguiente manera: niños de 7 a 10 años, adolescentes de 11 a 19 años, adultos jóvenes de 20 a 44 años, adultos medios de 45 a 59 años y adultos mayores de 60 años en adelante.

Variables

Se preparó un cuestionario en donde se preguntó como variables independientes el género, el nivel educativo y el estrato clasificando el 1 y el 2 como bajo, el 3 como medio y el 4, 5 y 6 como alto. Se clasificaron las variables dependientes (criterio de investigadores) como: El estado de ánimo (feliz, normal o triste). Para conocer la red de apoyo se preguntó: 'Cuando tiene problemas recibe apoyo de...' (11), clasificándola así: Red familiar: padres, hermano, hijo, abuelos. Red social: indagando por amigo, trabajo, profesores, comunidad (grupos comunitarios, servicios de salud o médicos y sacerdotes o pastores). Apoyo derivado por actividades individuales tales como deporte, estudio y el apoyo derivado de la percepción de Dios y de uno mismo. Los datos se redujeron a casi siempre ( 2 puntos), a veces (un punto) y casi nunca (0 puntos).

\section{Medidas}

Se plantearon 16 preguntas de apoyos, siendo 32 puntos (apoyo en todo) el puntaje máximo posible. Para seleccionar la red de apoyo alta y baja se obtuvo la media de los datos de apoyo y se aplicaron dos desviaciones estándar; se consideró red baja a la población que se situó en el cuartil inferior red media a la que se situó en los cuartiles medio inferior y medio superior y red alta a la que ubicó en el cuartel superior. Se obtuvo el OR entre los que se percibieron felices y tristes con red alta y baja en los ciclos vitales según variables independientes. 
Manejo estadístico

Las encuestas se transcribieron a una base de datos en hoja Excel y se cruzaron los datos con el programa Epi Info 2000. Se obtuvieron las regresiones logísticas incondicionales de las variables independientes confrontadas con los felices y los tristes y la red de apoyo alta con la baja.

Prueba piloto

Se entrenó a cinco promotoras de salud en la forma como debían hacer las preguntas y se hizo una prueba en 20 personas de distintas edades en Bogotá confirmando que ninguna se sintió incomoda por las preguntas y asegurando que contestaron con la verdad. No se notaron dificultades ni dudas para entender la encuesta. Cada encuesta tomó menos de cinco minutos.

Recolección de la información

Encuestadores (promotores) previamente entrenados aplicaron las encuestas entre los años 2002 y 2003, en Bogotá y en el municipio rural de Ubaté. A través de conocidos de estudiantes de una facultad de medicina, se completaron las encuestas en población de otros estratos. A cada miembro de familia seleccionado para la encuesta se le explicó la encuesta, cómo se mantendría la confidencialidad y la voluntariedad de participar. En el caso de los niños y jóvenes, los padres autorizaron su participación.

\section{RESULTADOS}

La edad media de la población estudiada fue de 30.7 años (DS de 21,6) la mediana por edad fue de 21 años y la moda de edad fue 9 años.

La población se repartió cronológicamente siguiendo el criterio de los ciclos vitales individuales como niños, adolescentes, adultos jóvenes, adultos maduros y adultos mayores (Tabla 1). Se encontró por cada triste tres felices.

La Tabla 2 muestra cómo la percepción del ánimo va cambiando de acuerdo a la edad; la felicidad va disminuyendo y la percepción de tristeza aumentado en la medida que se vive más. Pone en evidencia cómo los apoyos van cambiando en prevalencia a lo largo de los ciclos vitales, siendo el apoyo de Dios el más alto en promedio durante todos los ciclos (91\%), seguido de uno mismo $(77 \%)$, amigo (60 \%), madre (57\%), hermano (50\%) y servicios de salud (49\%). 
Tabla 1. Características sociodemográficas y de estado

\begin{tabular}{lrr}
\multicolumn{2}{c}{ de ánimo de población en Bogotá y Ubaté } \\
\hline \multicolumn{1}{c}{ Variable } & $N^{\circ}$ & $\%$ \\
\hline Sexo & 3572 & 49 \\
Mujer & 3763 & 51 \\
Hombre & 1119 & 15 \\
Ciclos vitales & 1098 & 15 \\
Más de 60 & 1631 & 22 \\
De 45 a 59 & 2222 & 30 \\
De 20 a 44 & 1247 & 17 \\
De 11 a 19 & & \\
De 7 a 10 & 5286 & 72 \\
Estrato & 1417 & 19 \\
Bajo & 638 & 9 \\
Medio & & \\
Alto & 4943 & 67 \\
Procedencia & 2400 & 33 \\
Urbano & & \\
Rural & 3413 & 47 \\
Nivel educativo & 2934 & 41 \\
Primaria & 887 & 12 \\
Secundaria & & \\
Universidad & 2259 & 31 \\
Estado de ánimo & 4314 & 59 \\
Feliz & 762 & 10 \\
$\quad$ Normal & & \\
Triste &
\end{tabular}

Tabla 2. Frecuencias de percepción de ánimo y apoyos a través del ciclo vital individual de población de Bogotá y Ubaté encuestada entre el 2002 y 2003

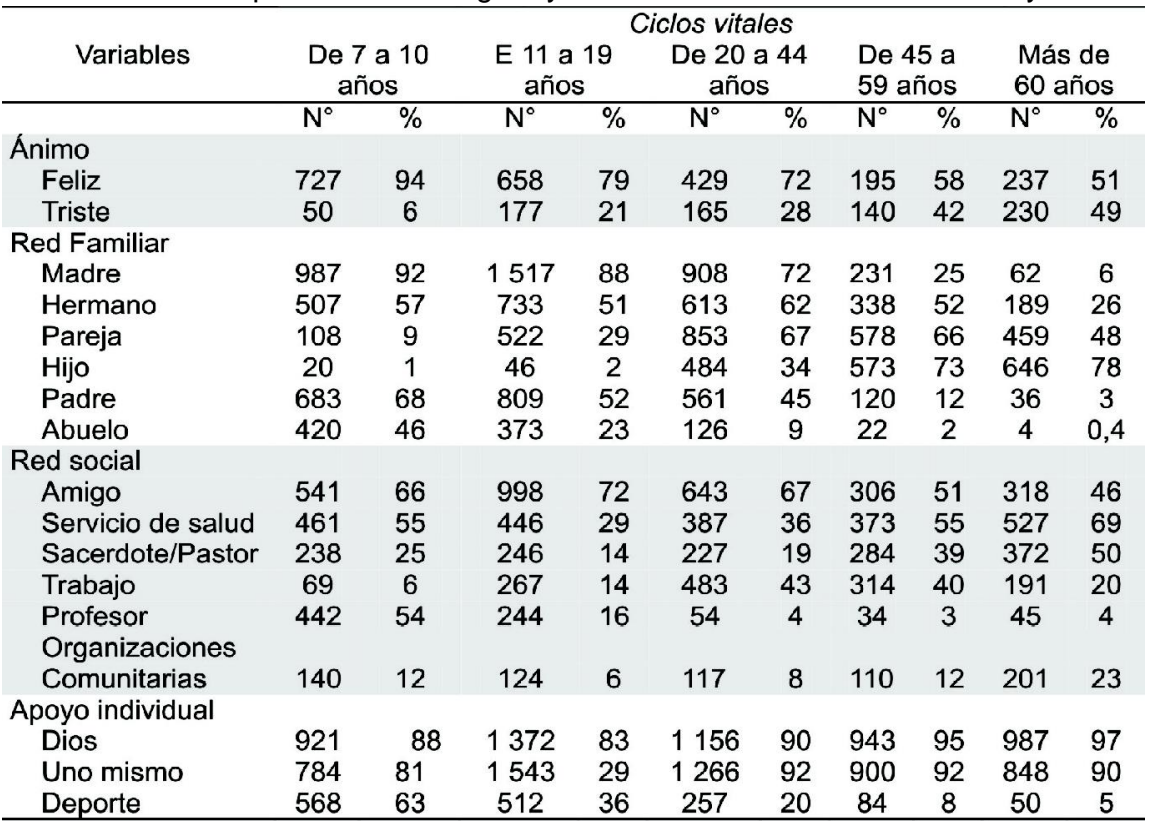


Los hombres en este estudio son más felices (Tabla 3), así como la población urbana, el estrato alto que el bajo (sin tendencia). Se observa tendencia entre tener más red de apoyo y felicidad (más red más feliz), así como con los ciclos vitales (a más joven más feliz) hasta los 45 años cuando de ahí en adelante no existen diferencias. El nivel educativo no estableció diferencias.

En cuanto al comportamiento de las redes de apoyo la media fue de 15.4 puntos, de 0 a12 puntos fueron los rangos del cuartel inferior y de 19 a 36 puntos los rangos del cuartel superior. Se observó una tendencia a tener redes altas en torno al ánimo (más feliz más red) y con la edad (entre más joven más red de apoyo). El género, ser urbano o rural o el estrato económico no establece diferencias. Con el nivel de estudio se observa diferencias significativas sin tendencia.

Tabla 3. Resultados de las razones de riesgo luego del análisis de regresión logística de los felices y los tristes con las variables demográficas, de apoyo, de ciclos vitales y estudio en una población de Bogotá y Ubaté entre el 2002 y el 2003

\begin{tabular}{|c|c|c|c|c|c|c|c|c|}
\hline \multirow{2}{*}{ Variables } & \multicolumn{2}{|c|}{ Feliz } & \multicolumn{2}{|c|}{ Triste } & \multirow[t]{2}{*}{ OR } & \multicolumn{2}{|c|}{ IC 95\% } & \multirow[t]{2}{*}{ Valor $\mathrm{P}$} \\
\hline & $\mathrm{N}^{\circ}$ & $\%$ & $\mathrm{~N}^{\circ}$ & $\%$ & & $\mathrm{LI}$ & LS & \\
\hline \multicolumn{9}{|l|}{ Demográficas } \\
\hline Mujer & 1120 & 71 & 469 & 30 & \multirow[b]{2}{*}{0,70} & \multirow[b]{2}{*}{0,6} & \multirow[b]{2}{*}{0,8} & \multirow{2}{*}{0,001} \\
\hline Hombre & 1134 & 80 & 293 & 21 & & & & \\
\hline Urbano & 1687 & 80 & 416 & 20 & \multirow{2}{*}{2,50} & \multirow{2}{*}{2,0} & \multirow{2}{*}{3,3} & \multirow{2}{*}{0,000} \\
\hline Rural & 572 & 62 & 346 & 38 & & & & \\
\hline Estrato medio & 506 & 80 & 122 & 19 & \multirow{2}{*}{1,02} & \multirow{2}{*}{0,7} & \multirow{2}{*}{1,3} & \multirow{2}{*}{0,857} \\
\hline Estrato bajo & 1479 & 71 & 596 & 19 & & & & \\
\hline Estrato alto & 274 & 86 & 44 & 14 & \multirow{2}{*}{1,60} & \multirow{2}{*}{1,0} & \multirow{2}{*}{2,4} & \multirow{2}{*}{0,016} \\
\hline Estrato bajo & 1479 & 71 & 596 & 29 & & & & \\
\hline \multicolumn{9}{|l|}{ Apoyos } \\
\hline Red de apoyo media & 1040 & 77 & 307 & 23 & \multirow[b]{2}{*}{2,95} & \multirow[b]{2}{*}{2,4} & \multirow{2}{*}{3,6} & \multirow{2}{*}{0,000} \\
\hline Red de apoyo baja & 437 & 54 & 369 & 46 & & & & \\
\hline Red de apoyo alta & 772 & 90 & 80 & 9 & \multirow{2}{*}{6,60} & \multirow{2}{*}{5,0} & & \\
\hline Red de apoyo baja & 437 & 54 & 369 & 46 & & & 9,0 & 0,000 \\
\hline Ciclos vitales & & & & & & & & \\
\hline De 45 a 59 años & 195 & 58 & 140 & 42 & & & 17 & 0204 \\
\hline De 60 o más años & 237 & 51 & 230 & 49 & 1,20 & 0,9 & 1,7 & 0,204 \\
\hline De 20 a 44 años & 429 & 72 & 165 & 28 & 160 & 12 & 23 & 0001 \\
\hline De 60 o más años & 237 & 51 & 230 & 49 & 1,60 & 1,2 & 2,3 & 0,001 \\
\hline De 11 a 19 años & 658 & 79 & 177 & 21 & 370 & 27 & & 0 \\
\hline De 60 o más años & 237 & 51 & 230 & 49 & 3,70 & 2,7 & 5,0 & 0,000 \\
\hline De 7 a 10 años & 727 & 94 & 50 & 6 & 121 & 86 & 18 & ח \\
\hline De 60 o más años & 237 & 51 & 230 & 49 & 12,4 & 8,6 & 18 & 0,000 \\
\hline Estudio & & & & & & & & \\
\hline Secundaria & 734 & 75 & 242 & 25 & 0 & 00 & 11 & $055 ?$ \\
\hline Primaria & 1194 & 74 & 425 & 25 & 1,10 & 0,8 & 1,4 & 0,553 \\
\hline Universitaria & 315 & 82 & 69 & 18 & 120 & 08 & 18 & 0310 \\
\hline Primaria & 1194 & 74 & 425 & 18 & & & & \\
\hline
\end{tabular}

Casos incluidos: 2945 
Tabla 4. Resultados de las razones de riesgo luego del análisis de regresión logística de los apoyos altos y los apoyos bajos con las variables demográficas, de ánimo de ciclos vitales y estudio de una población de Bogotá y Ubaté entre el

\begin{tabular}{|c|c|c|c|c|c|c|c|c|}
\hline \multirow{2}{*}{ Variables } & \multicolumn{2}{|c|}{ Red Alta } & \multicolumn{2}{|c|}{ Red Baja } & \multirow[t]{2}{*}{ OR } & \multicolumn{2}{|c|}{ IC $95 \%$} & \multirow[t]{2}{*}{ Valor $\mathrm{P}$} \\
\hline & $\mathrm{N}^{\circ}$ & $\%$ & $\mathrm{~N}^{\circ}$ & $\%$ & & LI & LS & \\
\hline \multicolumn{9}{|l|}{ Demográficas } \\
\hline Hombre & 811 & 44 & 1051 & 56 & \multirow{2}{*}{1,12} & \multirow{2}{*}{0,98} & \multirow{2}{*}{1,29} & \multirow{2}{*}{0,092} \\
\hline Mujer & 831 & 42 & 1130 & 58 & & & & \\
\hline Urbano & 1179 & 45 & 1419 & 55 & \multirow{2}{*}{1,09} & \multirow{2}{*}{0,92} & \multirow{2}{*}{1,30} & \multirow{2}{*}{0,299} \\
\hline Rural & 470 & 38 & 762 & 62 & & & & \\
\hline Estrato bajo & 1127 & 40 & 1640 & 59 & \multirow{2}{*}{0,92} & \multirow{2}{*}{0.75} & \multirow{2}{*}{1,12} & \multirow{2}{*}{0,435} \\
\hline Estrato medio & 349 & 48 & 379 & 52 & & & & \\
\hline Estrato bajo & 1127 & 40 & 1640 & 60 & \multirow{2}{*}{0,82} & \multirow[b]{2}{*}{0,62} & \multirow{2}{*}{1,08} & \multirow[b]{2}{*}{0,178} \\
\hline Estrato alto & 173 & 52 & 160 & 48 & & & & \\
\hline \multicolumn{9}{|l|}{ Ánimo } \\
\hline Feliz & 772 & 64 & 437 & 36 & \multirow{2}{*}{6,05} & \multirow{2}{*}{4,56} & \multirow{2}{*}{8,03} & \multirow{2}{*}{0,000} \\
\hline Triste & 80 & 18 & 369 & 82 & & & & \\
\hline Normal & 795 & 37 & 1373 & 63 & \multirow{2}{*}{2,36} & \multirow{2}{*}{1,81} & & \\
\hline Triste & 80 & 18 & 369 & 82 & & & 3,08 & 0,000 \\
\hline Ciclos vitales & & & & & & & & \\
\hline De 45 a 59 años & 191 & 36 & 332 & 63 & & & & \\
\hline De 60 o más años & 142 & 24 & 442 & 76 & 1,46 & 1,11 & 1,94 & 0,0072 \\
\hline De 20 a 44 años & 399 & 50 & 404 & 50 & & & & \\
\hline De 60 o más años & 142 & 24 & 442 & 76 & 1,90 & 1,45 & 2,50 & 0,000 \\
\hline De 11 a 19 años & 451 & 38 & 732 & 62 & & & & \\
\hline De 60 o más años & 142 & 24 & 442 & 76 & 1,34 & 1,03 & 1,75 & 0,026 \\
\hline De 7 a 10 años & 458 & 63 & 267 & 37 & & & & \\
\hline De 60 o más años & 142 & 24 & 442 & 76 & 3,60 & 2,77 & 4,68 & 0,000 \\
\hline Estudio & & & & & & & & \\
\hline Secundaria & 585 & 39 & 917 & 61 & & 0.64 & 0.96 & 0.016 \\
\hline Primaria & 780 & 43 & 1047 & 57 & 0.7 & 0.64 & 0.96 & 0,016 \\
\hline Universitaria & 281 & 63 & 165 & 37 & & & & \\
\hline Primaria & 780 & 43 & 1047 & 57 & 3,04 & 2,28 & 4,05 & 0,000 \\
\hline
\end{tabular}

\section{DISCUSIÓN}

La presente investigación tiene algunas limitaciones propias del diseño y de los encuestados: dado que no se trata de un formulario autodiligenciado existe alguna posibilidad de que no contesten con la verdad. Sin embargo, en la prueba piloto no se encontró razón de los encuestados para no ser sinceros. Aun así, y en particular, con los niños (7 a 9 años), ante la pregunta de si ha recibido apoyo del hijo, 20 respuestas evidenciaron error de información (si han recibido apoyo de hijos Tabla 2).

Es evidente que medir la felicidad no es fácil pero si es necesario para la promoción de la salud y el bienestar (4). La percepción de felicidad depende del contexto cultural del individuo (12) pero sin importar el sitio, siempre es acompañada por experiencias positivas. El estudio de Siahpush (1) propone medir la prevalencia del último mes de la percepción de felicidad. Este trabajo, 
la prevalencia de percepción del momento, lo cual limita la confiabilidad, pero como lo indica Ballas si no ocurre algún suceso fuera de lo común en la vida del encuestado (10) lo que conteste es lo esperado y se repetirá durante el ciclo vital en que esté. Lo cierto es que medir la felicidad es un indicador necesario que se debe promover y de hecho ya algunos autores lo proponen para identificar la enfermedad mental (13).

Esta investigación está dirigida a identificar la relación entre los apoyos del ser humano y la percepción de la felicidad y ahí la familia cobra singular importancia (14). El rol de apoyo de cada miembro indagado varía de acuerdo al ciclo vital, siendo el apoyo materno el más notorio de todos los acá indagados en los niños y adolescentes (Tabla 2) apoyo que se ve limitado por la inevitable desaparición materna que ocurrirá en general en la adultez de la vida de los apoyados (de los 45 a 59 años) pero que, sin embargo, alcanza una prevalencia (promediando percepción de apoyo en los ciclos) de $57 \%$. En cambio, el apoyo de un hermano puede mantenerse por más tiempo (todos los ciclos) pero con menos intensidad que al alcanzado por las madres (promedio de $50 \%$ a lo largo de la vida). Similar es el comportamiento del apoyo de un amigo (15) que está presente en cualquier etapa de la vida y por ello supera en el promedio incluso al promedio de la mamá (60\%). La pareja (44 \%) y los hijos (38\%) no alcanzan los promedios más altos (que son Dios, uno mismo y un amigo) porque "entran" a figurar más tarde (a partir de los 20 a 44 años). Sin embargo, la búsqueda de la pareja (16) es considerada un evento que hace feliz a las personas, especialmente si se enamoran (17) y que sucede más en la etapa de 20 a 44 años, cuando se alcanza la prevalencia más alta de este apoyo (67\%). Los hijos a su vez pasan a apoyar "fuerte" a partir de los 60 años (Tabla 2). Los padres apoyando con una tendencia similar a las madres no alcanzan a la misma en frecuencias. Los abuelos como apoyos disminuyen luego de la infancia, probablemente por su desaparición biológica.

El apoyo derivado de creer en Dios (promedio $91 \%$ de apoyo) es ciertamente muy importante para los seres humanos (18). El creyente es más comprometido con su vida y probablemente establece una relación directa con Dios que no amerita intermediaros como sacerdotes o pastores (que brindan en este estudio un apoyo promedio del $29 \%$ ). Interesante ha sido observar cómo los servicios de salud con una prevalencia del $49 \%$ son de los más frecuentes de apoyo ya que, sin ser en ninguna etapa el más frecuente, son por su presencia permanente en toda la vida bien percibidos. El trabajo si bien es importante, es evidente que en esta población no es un apoyo muy frecuente (media de) $25 \%$ ). Tal vez porque aún estamos lejos de trabajar en lo que gusta 
(se trabaja en lo que se puede), situación que hace que no sea muy clara la relación entre trabajo y felicidad (19). Los profesores y las organizaciones comunitarias ocupan las prevalencias más bajas de apoyo en esta población (16\% y $12 \%$ respectivamente).

Muy importante es la autoestima como apoyo (20) y es fuerte la relación con la felicidad $(5,6)$. La prevalencia media a lo largo de la vida de creer en sí mismo está situada en este estudio en $77 \%$ siendo la etapa de la adolescencia donde dicha percepción se ve sometida a su menor prevalencia (Tabla 2) cuando desciende (29\%) para volver a ascender en las siguientes etapas - crisis de la adolescencia - (21). ¿Qué implicaciones tiene cuando no asciende? Muy probablemente ha de tener una relación con la infelicidad (22) siendo por todo esto la adolescencia una etapa clave para este apoyo (5).

Esta investigación encontró una fuerte relación entre felicidad y redes sociales de apoyo (Tabla 3) incluso con tendencia bien marcada (OR de 2.95 y de 6.6 respectivamente) situación coherente con los resultados de investigadores de áreas económicas (23) en donde han encontrado que son los apoyos sociales más importante para sentirse feliz que la condición económica (comparar estratos y red de apoyo en Tabla 3) resultados discutibles al comparar el meta análisis de Howell (24) pero que confirman las conclusiones de North (8), Myers (15) y Diener (16). La Tabla 3 evidencia también la tendencia entre más años a ser menos feliz situación similar a la expuesta por Bjørnskov (25) y cuya explicación puede ser la disminución de los apoyos sociales en la medida que la edad avanza (Tabla 4). Esta investigación ofrece resultados diferentes a los de Myers (15) en cuanto a la relación entre felicidad y género (Tabla 3 donde hombres son más felices) y si bien coincide en que el estudio influye (universitarios comparados con primaria con OR de 3.04 en Tabla 3 ) y que se confirma por la relación entre las redes de apoyo (Tabla 4) no se observan diferencias entre estudio de secundaria y primaria (a más nivel de estudio más felicidad y más apoyo).

En conclusión, esta investigación confirma la relación entre la felicidad y las redes de apoyo sociales conclusión similar a la Fowler (9) que demostró cómo la cercanía con los amigos contribuye a sentirse feliz. Promueve la necesidad de fortalecer las relaciones sociales siendo los niños claves por ser la etapa donde se construyen la mayor cantidad de apoyos. Implica la necesidad de abordar terapéuticamente enfoques como el propuesto por Lee (26) y Clonninger (27) correspondiendo a la salud mental (salud emocional), siendo por sobre todo indispensable enseñarles a los niños a manejar los conflictos 
(10) para no romper con los demás (y no disminuir la red de apoyo), así como enseñar a manejar los malos momentos de la vida (28). Así mismo, y en los otros ciclos de la vida, se ha de promover compartir con los demás y no "romper"; al hacerlo se fortalece la salud del individuo y el bienestar (29). La medicina tiene un importante campo de acción al incorporar a la felicidad en la consulta (30) así como el fomento del contacto de los pacientes con los demás; al hacerlo está promoviendo el bienestar que es el fin de tener salud *

Agradecimientos: A los estudiantes del postgrado de Salud Familiar de la promoción del 2002 de la Fundación Universitaria Juan N Corpas que ubicaron la población de Ubaté a través de la Administradora del Régimen Subsidiado de Cafam y del pregrado de medicina (cátedra de salud familiar del 2003) de la fundación universitaria Juan N Corpas. A Innosalud Ltda., entidad que aportó recursos para la ejecución de esta investigación.

\section{REFERENCIAS}

1. Siahpush M, Spittal M, Singh GK. Happiness and life satisfaction prospectively predict selfrated health, physical health, and the presence of limiting, long-term health conditions. Am J Health Promot. 2008; 1:18-26.

2. Hsee CK, Tang JN. Sun and water: on a modulus-based measurement of happiness. Emotion. 2007; 7:213.

3. Chida Y, Steptoe A. Positive psychological well-being and mortality: a quantitative review of prospective observational studies. Psychosom Med. 2008; 70:741-56.

4. Barak Y, Achiron A. Happiness and neurological diseases. Expert Rev Neurother. 2009; 9:445-59.

5. Caprara GV, Steca P, Gerbino M, Pacielloi M, Vecchio GM. Looking for adolescents' wellbeing: self-efficacy beliefs as determinants of positive thinking and happiness. Epidemiol Psichiatr Soc. 2006; 15(1):30-43.

6. Delamothe T. Happiness. BMJ 2005; 331:1489-1490.

7. Kossinets G, Watts DJ. Empirical Analysis of an Evolving Social Network. Science. 2006; $311,88$.

8. North RJ, Holahan CJ, Moos RH, Cronkite RC Family support, family income, and happiness: a 10-year perspective. J Fam Psychol. 2008; 22:475-83.

9. Fowler J, Christakis N. Dynamic spread of happiness in a large social network: longitudinal analysis over 20 years in the Framingham Heart Study. BMJ 2008; 337:2338.

10. Ballas D, Dorling D. Measuring the impact of major life events upon happiness. Int $J$ Epidemiol. 2007; 36:1244-52.

11. Arias L, Smilkstein G. Dra.-A-tree: An instrument to assess family function. Dept of Family Medicine University of Louisville.USA; 1992.

12. Carlisle S, Hanlon P. Well-being and consumer culture: a different kind of public health problem? Health Promot Int. 2007; 22(3):261-8.

13. Bray I, Gunnell D. Suicide rates, life satisfaction and happiness as markers for population mental health. Soc Psychiatry Psychiatr Epidemiol. 2006; 41(5):333-7.

14. North RJ, Holahan CJ, Moos RH, Cronkite RC. Family support, family income, and happiness: a 10-year perspective. J Fam Psychol. 2008; 22(3):475-83. 
15. Myers DG. The funds, friends and faith of happy people. Am Psychol. 2000; 55(1):56-67. 16. Diener E, Seligman ME. Very happy people. Psychol Sci. 2002; 13(1):81-4.

17. Pettijohn TF 2nd, Pettijohn TF. Perceived happiness of college students measured by Maslow's hierarchy of needs. Psychol Rep. 1996; 79(3 Pt 1):759-62.

18. Ellison CG. Religious involvement and subjective well-being. J Health Soc Behav. 1991; 32(1):80-99.

19. Kaliterna LL, Prizmic LZ, Zganec N. Quality of life, life satisfaction and happiness in shiftand non-shiftworkers. Rev Saude Publica. 2004; 38 Suppl:3-10.

20. Neto F. Personality predictors of happiness. Psychol Rep. 2001; 88(3 Pt 1):817-24.

21. Guerrero O. ¿Existe la crisis de la adolescencia? Acta pediátrica de México. 2003; 24 (1).

22. Francois $L$. ¿Para qué una identidad? o el embrollo de las identificaciones y su reorganización en la adolescencia. Psicoanálisis Ap de Ba. 2001; 23 (2).

23. Helliwell JF, Putnam RD The social context of well-being. Philos Trans R Soc Lond B Biol Sci. 2004; 359(1449):1435-46.

24. Howell RT, Howell CJ. The relation of economic status to subjective well-being in developing countries: a meta-analysis. Psychol Bull. 2008; 134 pp536-60.

25. Bjørnskov C. Healthy and happy in Europe? On the association between happiness and life expectancy over time. Soc Sci Med. 2008; 66.pp1750-9.

26. Lee Duckworth A, Steen TA, Seligman ME. Positive psychology in clinical practice. Annu Rev Clin Psicol.2005;1:629-51.

27. Cloninger $C R$. The science of well-being: an integrated approach to mental health and its disorders. World Psychiatry. 2006; 5(2):71-6.

28. Reich JW, Zautra A. Life events and personal causation: some relationships with satisfaction and distress. J Pers Soc Psychol. 1981; 41(5):1002-12.

29. Graney MJ. Happiness and social participation in aging. J Gerontol. 1975; 30(6):701-6.

30. Hershberger PJ. Prescribing happiness: positive psychology and family medicine. Fam Med. 2005; 37(9):630-4. 